\title{
A search for evidence of irradiation in Centaurus X-4 during quiescence ${ }^{\star}$ (Research Note)
}

\author{
P. D’Avanzo ${ }^{1,2}$, T. Muñoz-Darias ${ }^{3}$, J. Casares ${ }^{3}$, I. G. Martínez-Pais ${ }^{3}$, and S. Campana ${ }^{1}$ \\ 1 INAF - Osservatorio Astronomico di Brera, via Bianchi 46, 23807 Merate (Lc), Italy \\ e-mail: paolo.davanzo@brera.inaf.it \\ 2 Università degli Studi dell'Insubria, Dipartimento di Fisica e Matematica, via Valleggio 11, 22100 Como, Italy \\ 3 Instituto de Astrofisica de Canarias, 38200 La Laguna, Tenerife, Spain
}

Received 11 July 2006 / Accepted 5 September 2006

\section{ABSTRACT}

\begin{abstract}
Aims. We present a study of the neutron star X-Ray Transient Cen X-4. Our aim is to look for any evidence of irradiation of the companion with a detailed analysis of its radial velocity curve, relative contribution of the donor star and Doppler tomography of the main emission lines.

Methods. To improve our study all our data are compared with a set of simulations that consider different physical parameters of the system, like the disc aperture angle and the mass ratio.

Results. We conclude that neither the radial velocity curve nor the orbital variation of the relative donor's contribution to the total flux are affected by irradiation. On the other hand, we do see emission from the donor star at $\mathrm{H} \alpha$ and $\mathrm{HeI} \lambda 5876$ which we tentatively attribute to irradiation effects. In particular, the $\mathrm{H} \alpha$ emission from the companion is clearly asymmetric and we suggest is produced by irradiation from the hot-spot. Finally, from the velocity of the HeI $\lambda 5876$ spot we constrain the disc opening angle to $\alpha=7^{\circ}-14^{\circ}$.
\end{abstract}

Key words. accretion, accretion disks - binaries: close - stars: individual: Cen X-4 - stars: neutron

\section{Introduction}

Soft X-Ray Transients (SXRTs), a subclass of Low Mass X-Ray Binaries, are interacting binary systems which alternate short periods (weeks to months) of high X-ray luminosity with long (several years) intervals of quiescence. During quiescence, such transient systems, are very faint in X-rays $\left(10^{32}-10^{33} \mathrm{erg} \mathrm{s}^{-1}\right)$ and their optical luminosity drops by as much as 6-7 mag, giving a unique opportunity for the study of companion stars (see Campana et al. 1998, for a review).

Cen X-4 is a well-known neutron star X-ray transient, from which two bright X-ray outburst were detected in 1969 (Conner et al. 1969) and in 1979 (Kaluzienski et al. 1980). From then on it remained in the quiescent state. With an evolved $0.3 M_{\odot}, \mathrm{K} 3-\mathrm{K} 7$ star (Shahbaz et al. 1993; Torres et al. 2002; D'Avanzo et al. 2005), Cen X-4 is one of the brightest SXRTs in quiescence, with $V=18.7$ and low interstellar absorption $\left(A_{V}=0.3 \mathrm{mag}\right)$. A $15.1 \mathrm{~h}$ orbital period was determined from the sinusoidal variation of the optical light curve (Cowley et al. 1988; Chevalier et al. 1989; McClintock \& Remillard 1990). Both the optical spectrum and emission line maps obtained with the Doppler tomography technique (Marsh \& Horne 1988) show clear evidence of disc emission (Torres et al. 2002; D'Avanzo et al. 2005).

In general, the effect of heating of the secondary star leads to a change in absorption lines strength, with the consequent effect to move the effective centre of the secondary away from the centre of mass of the star. This causes phase-dependent distortions

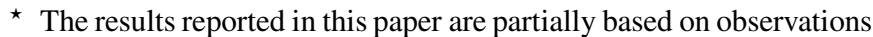
carried out at ESO, La Silla, Chile (67.D-0116). of the radial velocity from a sinusoidal fit, leading to incorrect determinations of the radial velocity amplitude.

In our previous work on Cen X-4 evidence of irradiation was proposed in light of a residual emission of $\mathrm{H} \alpha$ and $\mathrm{HeI}$ from the companion star. We present here a search for evidence of irradiation in Cen X-4, comparing our observations presented in D'Avanzo et al. (2005) with the simulations obtained using the code presented in Muñoz-Darias et al. (2005).

\section{The radial velocity curve of Cen X-4}

In D'Avanzo et al. (2005) we presented the results of the first extensive and contiguous orbital coverage of Cen X-4. In Fig. 1, the radial velocity curve we obtained for Cen X-4 is shown, together with the residuals to the sinusoidal fit. To overcome any spurious effect, we have carefully corrected our spectra for any telescope flexure, using telluric lines. It is clear from Fig. 1 that there are deviations from the best fit; such deviations are of the order of a few $\mathrm{km} \mathrm{s}^{-1}$.

In order to understand if this behaviour could be considered as an indication of irradiation we have compared our observational result with a simulation obtained with the code developed by Muñoz-Darias et al. (2005). In this simulation we model the effect of irradiation in radial velocity curves derived from the Doppler shift of absorption lines formed by the companion. These features are quenched in the heated face of the donor and hence the observed $K$-velocity provides only an upper limit to the real $K_{2}$. This deviation roughly depends on the X-ray luminosity, the orbital separation and the opening disc angle $(\alpha)$ which partly obscures the companion. Here, we have only 


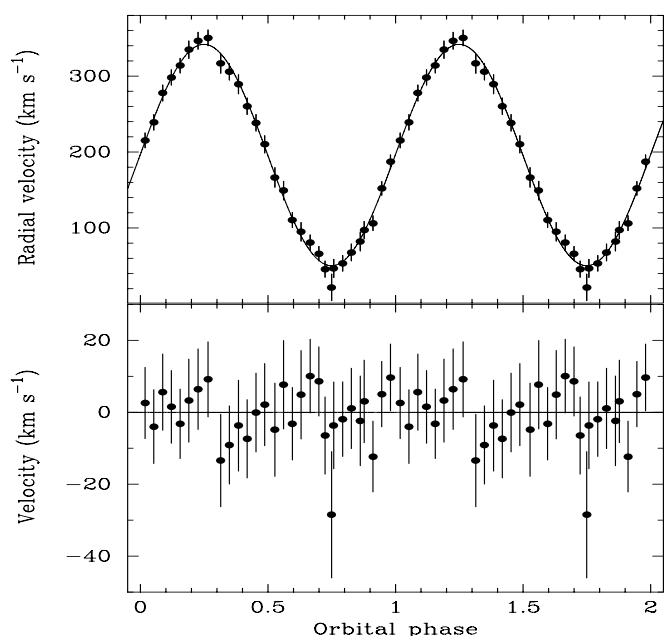

Fig. 1. Radial velocity curve for Cen X-4 (from D'Avanzo et al. 2005) obtained through cross-correlation of absorption lines in the range 5930-6750 ^ with late-type template stars. Two phases are shown for clarity. The best sine-wave fit is also shown. Residuals from the best fit are shown in the lower panel.

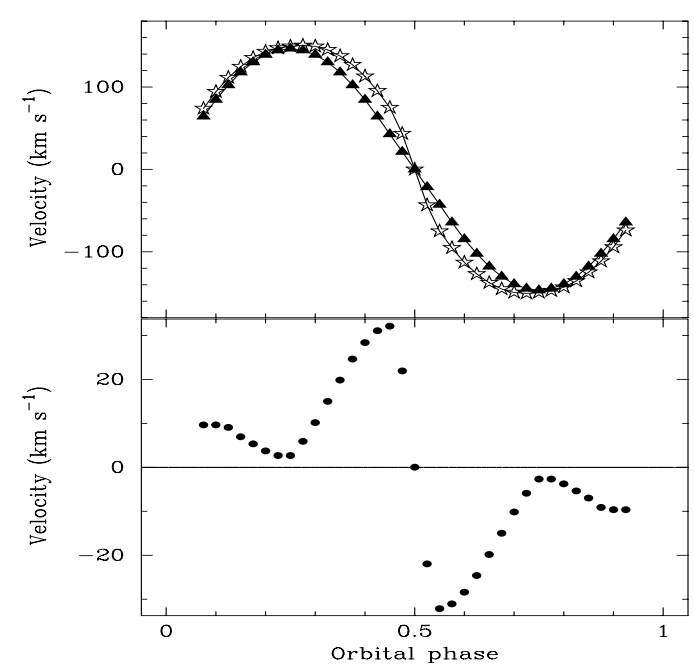

Fig. 2. Simulated radial velocity curves for Cen X-4 for the irradiated (stars) and non irradiated (triangles) case. Differences between the two models are shown in the lower panel.

considered the limit case in which the absorption lines are totally quenched on the irradiated regions of the donor star which are not shadowed by the disc.

We have produced two curves (both showed in Fig. 2), representing the irradiated and the non-irradiated case. Residuals are shown at the bottom of the figure. It is immediately clear that the main effect of irradiation from the neutron star is the rise in the velocity semi-amplitude, as shown. Such a behaviour is clearly observed in the radial velocity curves of systems where the secondary stars are exposed to irradiation from the compact object (like, e.g., Friend et al. 1990a,b).

In light of the results of our simulations, we can conclude that the radial velocity curve does not show any evidence of irradiation. The expected rise in the velocity between phases 0.25 and 0.75 (i.e. when the observer sees the inner face of the companion) is not present. Although deviations from the sinusoidal fit seem to be present in our observed curve these are significantly smaller (given the error bars) than predicted by the irradiation model, and most important, these deviations are in

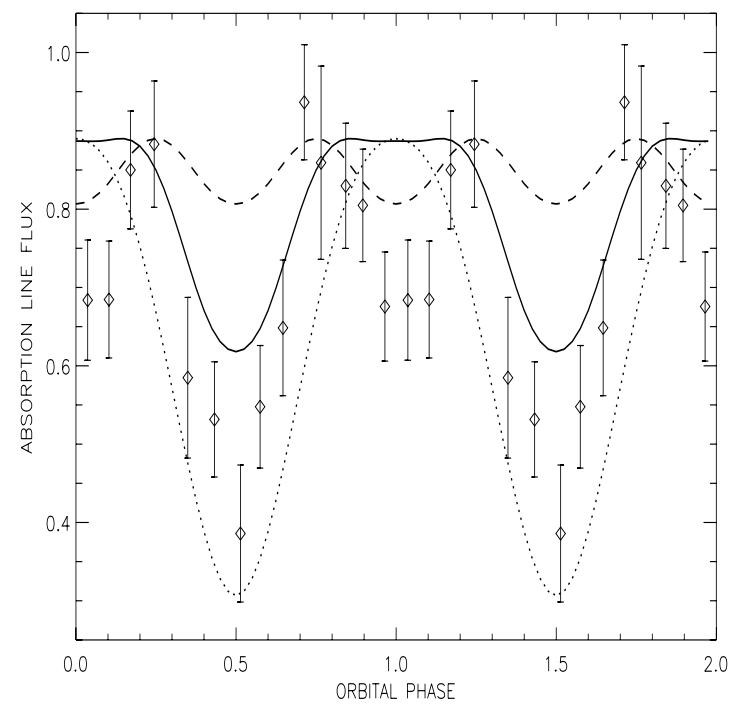

Fig. 3. Variations of the fractional contribution of the companion compared with synthetic models for $\alpha=0^{\circ}$ (dotted line), $7^{\circ}$ (solid line) and $14^{\circ}$ (dashed line).

the opposite direction to what is expected in an irradiation scenario.

\section{The optical light curve of the companion}

If the inner side of the secondary star is exposed to irradiation this would also affect the modulation of the contribution of the companion to the optical luminosity. In D'Avanzo et al. (2005) we determined the variation of the fractional contribution of light from the secondary (named factor $f$ ) for 30 phase bins. The result was that $f$ is not constant at all the orbital phases, but modulated with two unequal minima at phase 0 (i.e. at superior conjuction) and 0.5 and two nearly equal maxima at phases 0.25 and 0.75 (i.e. when the observer sees the sides of the companion with the maximum surface projected area). This behaviour is reminiscent of the classical ellipsoidal modulation (see e.g. McClintock \& Remillard 1990; Shahbaz et al. 1993) caused by the changing visibility of the tidally distorted companion as seen by the observer. However, we note the unusually large difference in depth between phases 0 and 0.5 , requiring an additional effect like, e.g. the heating of the inner hemisphere of the companion by X-ray irradiation from the neutron star. In order to investigate this scenario we compare the variation of the $f$ factor (coadded into 14 phase bins to increase the $\mathrm{S} / \mathrm{N}$ ratio) with a set of synthetic curves obtained with the code developed by Muñoz-Darias et al. (2005). This is presented in Fig. 3.

These simulations show the absorption line flux as function of the orbital phase for three different flaring angles $(\alpha)$. It is well established that the temperature of the outer layers of the companion can be upset by external heating (e.g. Brett \& Smith 1993), which removes the vertical temperature gradient of the heated star and quench the absorption lines within the irradiated regions. In order to compute our curves we have considered the limit case in which absorption lines are totally quenched (i.e. not present) on the irradiated regions of the companion (see also Shahbaz et al. 2000). As it is clear in the plot we cannot reconcile the models and the data for any value of $\alpha$. If the opening angle is low, the absorption line is quenched upon the whole irradiated hemisphere of the companion and hence the absorption line flux is very low at orbital phases close to 0.5 (dotted line). However, we can not reproduce the lower $f$ factor found for orbital phases 
around 0 . On the other hand, for $\alpha \geq 14^{\circ}$ (dashed line) the accretion disc completely screens out the companion star, the effect of irradiation disappears and we obtain the ellipsoidal modulation associated with the changing visibility of the tidally distorted companion star. If we consider the X-ray luminosity of $L_{\mathrm{X}} \sim 10^{32} \mathrm{erg} \mathrm{s}^{-1}$ reported in Campana et al. (2004) and the orbital parameters of the system, we estimate using our binary code that the effective temperature of the irradiated regions of the donor is only $0-30 \mathrm{~K}$ higher than in the non-irradiated regions. Hence, the donor's atmosphere should almost not be affected by heating and we expect an $f$ factor behaviour close to the latter case in which irradiation effects are not present. Therefore, as result of our simulations, the irradiation from the inner regions of the accretion disc does not appear to be responsible for the orbital behaviour of the $f$ factor.

\section{4. $\mathrm{H} \alpha$ and $\mathrm{Hel}$ emission from the companion}

In D'Avanzo et al. (2005) we presented Doppler maps of Cen X-4 showing clear evidence of residual $\mathrm{H} \alpha$ and HeI $\lambda 5876$ emission associated with the companion star. As opposed to $\mathrm{HeI} \lambda 25876$, the $\mathrm{H} \alpha$ spot is clearly offset with respect to the vertical $V_{y}$ axis. As reported in D'Avanzo et al. (2005) we have computed the centroids of these spots obtaining:

$\mathrm{H} \alpha:\left(V_{x}, V_{y}\right)=(34.1 \pm 11.8,97.7 \pm 11.8) \mathrm{km} \mathrm{s}^{-1}$

HeI 15876: $\left(V_{x}, V_{y}\right)=(10.3 \pm 11.8,122.8 \pm 11.8) \mathrm{km} \mathrm{s}^{-1}$.

This clearly demonstrates that the offset of the $\mathrm{H} \alpha$ spot is significant at $\sim 3-\sigma$ and it leads phase 0 by $0.05 \pm 0.03$ cycles. The S-wave component responsible for the $\mathrm{H} \alpha$ spot is clearly seen in the individual spectra. Therefore, in order to reduce the uncertainty in the position of the region of the companion emitting $\mathrm{H} \alpha$ photons we have performed a multi-Gaussian fit to the $\mathrm{H} \alpha$ profiles, coadded into 30 phase bins. Such operation enabled us to isolate the $\mathrm{H} \alpha$ component emitted from the companion for each phase bin, and to determine with good precision its centroid. As a result we obtained a sinusoid-like velocity curve, shown in Fig. 4, whose semi-amplitude (equal to $91.1 \pm 3.0 \mathrm{~km} \mathrm{~s}^{-1}$ ) is in agreement with the value of $V_{y}$ obtained from the Doppler map, but it is more precise and gives the velocity of the $\mathrm{H} \alpha$ emission region of the companion. Moreover, we also note that Fig. 4 shows also an indication of a phase shift (equal to $0.02 \pm 0.01$ ) which is in agreement to the shift in the $V_{x}$ direction of the $\mathrm{H} \alpha$ spot in the Doppler map.

Unfortunately the lower $\mathrm{S} / \mathrm{N}$ ratio of the HeI $\lambda 5876$ line does not enable us to do an accurate multi-Gaussian fit to isolate the companion component, so we used the position given from the Doppler map. In this case no phase/velocity shift is present in the $x$ direction.

In D'Avanzo et al. (2005) we measured an equivalent width for the $\mathrm{H} \alpha$ residual of $4.4 \pm 0.5 \AA$ which could not be explained only invoking chromospheric activity from the companion. Hence, we proposed that the $\mathrm{H} \alpha$ spot may be triggered by irradiation from the compact star (for details see D'Avanzo et al. 2005). This scenario predicts lower excitation lines to be formed at higher Roche lobe latitudes because of variable photoelectric absorption by the outer disc rim i.e. lower energy photons are more efficiently absorbed and hence lower excitation lines arise from regions close to the poles. This has been previously seen in the cataclysmic variable IP Peg (Harlaftis 1999; Morales-Rueda et al. 2000) and more recently in U Gem (Unda-Sanzana et al. 2006). This scenario, however, does not agree with our observed velocities for $\mathrm{H} \alpha$ and HeI emission. Moreover, with a velocity semi-amplitude of

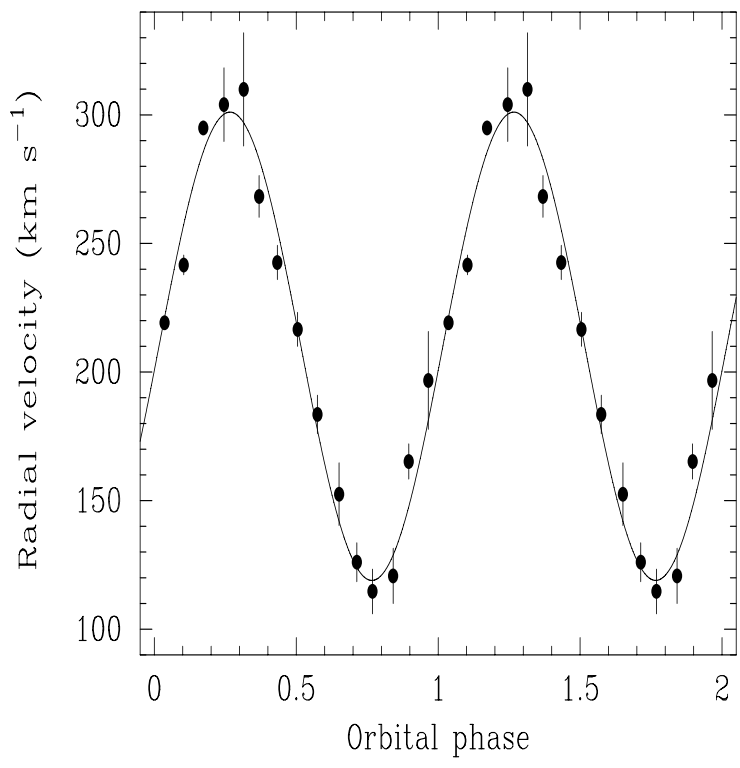

Fig. 4. Velocity of the $\mathrm{H} \alpha$ emission from the companion at different orbital phases. Two cycles are shown for clarity.

$91.1 \pm 3.0 \mathrm{~km} \mathrm{~s}^{-1}$, the $\mathrm{H} \alpha$ residual emission arises from the vicinity of the $\mathrm{L}_{1}$ point which must be efficiently shadowed from the UV photons by the disc. This, together with the asymmetric distribution of the $\mathrm{H} \alpha$ spot over the Roche lobe, points to the hotspot as a possible source of the $\mathrm{H} \alpha$ photoionising radiation.

\section{Discussion}

The discrepancies between the observed and the simulated radial velocity curve enable us to conclude that our radial velocity curve of Cen X-4 does not contain any evidence of the presence of an irradiated companion and that the measured semiamplitude of the curve must correspond to the "real" $\mathrm{K}_{2}$ velocity of the secondary star. This is also confirmed by new high quality UVES data at $7 \mathrm{~km} \mathrm{~s}^{-1}$ resolution (Casares et al. 2006, in preparation). Therefore, no "K-correction" seems to be necessary for this system.

The interpretation of the orbital modulation of the optical luminosity contribution of the companion is more trivial. We note here that the $f$-factor yields not the lightcurve of the companion star but the relative contribution to the total flux. Therefore, instead of neutron star irradiation, a more plausible explanation for the deep phase 0.5 minimum would simply be an increase in the disc brightness around this particular phase i.e. a non-uniform emissivity distribution which might trace azimutal variations of the disc density.

Neutron star irradiation seems to be ruled out also as responsible for the $\mathrm{H} \alpha$ emission from the companion. In fact, as we have seen in the previous section, the $\mathrm{H} \alpha$ residual arises from a region of the companion which should be affected by the shadow of the disc.

In light of this, hot-spot irradiation seems to be a natural alternative explanation for the intrinsic $\mathrm{H} \alpha$ emission from the companion, because in such case, no shielding is to be considered. In fact, as we see from our Doppler maps, bright anisotropies are present on the accretion disc. We propose that the $\mathrm{H} \alpha$ emission concentrates in the trailing side of the Roche lobe because the leading side is shielded by gas stream. Hotspot heating has been previously invoked to explain asymmetric emission patterns observed in several cataclysmic variables (e.g. 


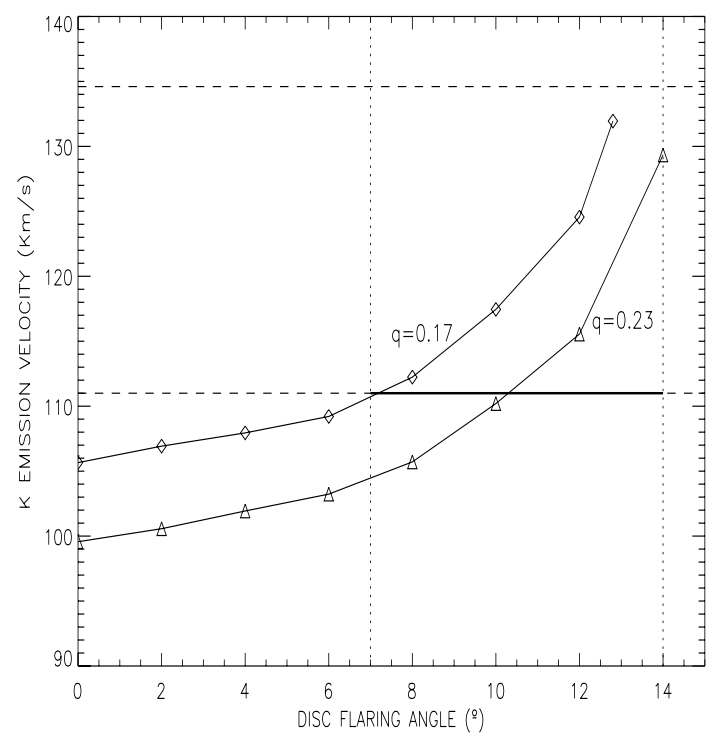

Fig. 5. Determination of the disc flaring angle in Cen X-4. The solid lines represent the result of our model for the considered mass ratio values $(q \sim 0.17$ and $q \sim 0.23)$. The dashed lines represent the value $K_{\mathrm{em}}=122.8 \pm 11.8 \mathrm{~km} \mathrm{~s}^{-1}$ measured from or Doppler maps. The possible disc flaring angle range is marked with a solid line.

in IX Vel, see Beuermann \& Thomas 1990). Furthermore, evidence for a hot-spot or splash point in Cen X-4 was presented by McClintock \& Remillard (1990) whereas our HeI Doppler images (Fig. 4 in D'Avanzo et al. 2005) also show evidence of bright spots coincident with the interaction between the outer disc rim and the gas stream. Alternatively, asymmetric heating may be amplified by the action of circulation currents induced through Coriolis forces (Davey \& Smith 1992; Martin \& Davey 1995). A direct consequence of hot-spot (if present) or, in general, anisotropy irradiation is the shift, in the $V_{x}$ direction, of the $\mathrm{H} \alpha$ emission region on the companion, exactly as observed.

On the contrary, the higher $V_{y}$ and the absence of any phase/velocity shift in the $x$ direction, should suggest that the HeI emission region on the companion lies in a region of the Roche Lobe that is out from the shadow of the disc and directly exposed to illumination by the neutron star. We can use this hypothesis to put some strong constraints on the aperture angle $\alpha$ of the accretion disc. To do this, we have used the K-correction values tabulated in Muñoz-Darias et al. (2005). In this work, the relation $K_{\mathrm{em}} / K_{2}$ is computed as function of the mass ratio ( $q=\frac{M_{1}}{M_{2}}$ ) for different values of $\alpha$. Where $K_{2}$ is the radial velocity associated with the mass center of the companion and $K_{\mathrm{em}}$ is the velocity of an emission line formed on the heated face of the donor. Since $q$ and $K_{2}$ are known for Cen X-4 we have used the observed $K_{\mathrm{HeI}}$ emission line velocity to constrain the value of $\alpha$. As is explained above, the low $\mathrm{S} / \mathrm{N}$ ratio of this line does not allow us to isolate the companion component and hence we have used the $K_{\mathrm{em}}=122.8 \pm 11.8 \mathrm{~km} \mathrm{~s}^{-1}$ obtained from the
Doppler map. In Fig. 5 we show that using this value of $K_{\mathrm{em}}$ and considering $0.17 \leq q \leq 0.23$ we directly obtain $\alpha \geq 7^{\circ}$. Moreover, if we take into account the maximum value of $\alpha$ to the companion be irradiated for this $q$, we can further constrain the opening disc angle between $7^{\circ} \leq \alpha \leq 14^{\circ}$ (Fig. 5).

\section{Conclusions}

We have examined the $\mathrm{H} \alpha$ and $\mathrm{HeI} \lambda 5876$ bright spots associated to the companion star in Cen X-4. The HeI spot is well centered on the line joining the two stars and we use its velocity to constrain the disc flaring angle to be $7-14^{\circ}$. On the other hand, the $\mathrm{H}_{\alpha}$ spot concentrates around the $\mathrm{L} 1$ point and is shifted towards the leading side of the Roche lobe. We tentatively explain this asymmetry through irradiation from the hot-spot and shielding by the gas stream.

We have also searched for evidence of irradiation effects in the radial velocity curve of the photospheric absorptions, by comparing our data with model simulations, but found none. This lead us to conclude that the donor's metallic lines are not affected by irradiation and hence the orbital parameters presented in previous papers (Torres et al. 2002; D’ Avanzo et al. 2005) are safe from bias.

Acknowledgements. P.D.A. thanks the Astrophysics Institute of the Canary Islands (IAC) for kind hospitality. S.C. and P.D.A. acknowledge the Italian Space Agency for financial support through the project ASI I/R/023/05. J.C. and P.D.A. acknowledge support from the Spanish Ministry of Science and Technology through the project AYA2002-03570.

\section{References}

Beuermann, K., \& Thomas, H. -C. 1990, A\&A, 230, 326

Brett, J. M., \& Smith, R. C. 1993, MNRAS, 264, 641

Campana, S., Colpi, M., Mereghetti, S., et al. 1998, A\&AR, 8, 279

Campana, S., Israel, G. L., Stella, L., et al. 2004, ApJ, 601, 474

Casares, J., et al. 2006, in prep.

Chevalier, C., Ilovaisky, S. A., van Paradijs, J., et al. 1989, A\&A, 210, 114

Conner, J. P., Evans, W. D., \& Belian, R. D. 1969, ApJ, 157, 157

Cowley, A. P., Hutchings, J. B., Schmidtke, P. C., et al. 1988, AJ, 95, 1231

D’Avanzo, P., Campana, S., Casares, J., et al. 2005, A\&A, 444, 905

Davey, S. C., \& Smith, R. C. 1992, MNRAS, 257, 476

Friend, M. T., Martin, J. S., Smith, R. C., \& Jones, D. H. P. 1990a, MNRAS, 246, 637

Friend, M. T., Martin, J. S., Smith, R. C., \& Jones, D. H. P. 1990b, MNRAS, 246,654

Harlaftis, E. T. 1999, A\&A, 346, L73

Kaluzienski, L. J., Holt, S. S., \& Swank, J. H. 1980, ApJ, 241, 779

Marsh, T. R., \& Horne, K. 1988, MNRAS, 235, 269

Martin, T. J., \& Davey, S. C. 1995, MNRAS, 275, 31

McClintock, J. E., \& Remillard, R. 1990, ApJ, 350, 386

Morales-Rueda, L., Marsh, T. R., \& Billington, I. 2000 MNRAS, 313, 454

Muñoz-Darias, T., Casares, J., \& Martínez-Pais, I. G. 2005, ApJ, 635, 502

Shahbaz, T., Naylor, T., \& Charles, P. A. 1993, MNRAS, 265, 655

Shahbaz, T., Groot, P., Phillips, S. N., et al. 2000, MNRAS, 314, 747

Torres, M. A. P., Casares, J., Martínez-Pais, I. G., \& Charles, P. A. 2002, MNRAS, 334, 233

Unda-Sanzana, E., Marsh, T. R., \& Morales-Rueda, L. 2006, MNRAS, 369, 805 